\title{
Finding Meaning in 4D STEM: Strategies for Extracting Targeted Specimen Information
}

Joanne Etheridge ${ }^{1}$, Wei Chao ${ }^{1}$, Espen Drath Bøjesen ${ }^{2}$, Bryan Esser ${ }^{1}$, Matus Krajnak ${ }^{3}$, Weilun Li $^{1}$, TC Petersen ${ }^{1}$ and Changlin Zheng $^{4}$

${ }^{1}$ Monash University, Melbourne, Victoria, Australia, ${ }^{2}$ Aarhus University, Aarhus C, Midtjylland, Denmark, ${ }^{3}$ Quanum Detectors Ltd., Harwell Oxford, England, United Kingdom, ${ }^{4}$ Fudan University, Shanghai, Shanghai, China (People's Republic)

Four-dimensional Scanning Transmission Electron Microscopy (4D-STEM) provides a two-dimensional spatial map of electron diffraction patterns, most commonly generated using a convergent beam. It has been known for well over half a century that convergent beam electron diffraction (CBED) patterns contain a wealth of information about the specimen, including local symmetry, structure factor amplitudes and phases, bonding, lattice parameters, specimen thickness, strain, and polarity. With the new generation of high speed, high dynamic range detectors, it is now possible to map and record the CBED pattern at each position of the electron probe in a STEM experiment, delivering huge and complex data sets.

In principle, almost all specimen information is contained within these powerful 4D-STEM data sets. The challenge is to find efficient ways to optimize and extract it. In this talk, we describe a variety of strategies for deriving meaningful information from this data. We consider how we can craft the incident electron wave field and scattering within the specimen to generate a scattered intensity distribution rich in the specimen information we want. We then tailor the detector geometry to detect this information at each probe position.

These strategies will be illustrated with applications to measuring the structure and defect structure of functional perovskites; surface composition and structure in metallic nanoparticles; three-dimensional reconstructions of nanoparticle shape; and compositional measurements and defect structures in semiconductor systems. We will also demonstrate a novel mechanism for generating atomic resolution image contrast that derives from the local symmetry within the specimen (Symmetry-STEM). Experiments were undertaken using a double-aberration-corrected FEI Titan ${ }^{3}$ 80-300 FEG-TEM fitted with a pixelated detector (the "electron microscope pixel array detector" or EMPAD based on the Cornell design [1]) and a high-speed CMOS-based FEI CETA camera.

\section{References}

[1] MW Tate et. al., Microscopy and Microanalysis 22 (2016) p. 237.

[2] JE acknowledges funding from the Australian Research Council grants DP150104483, DP160104679 and LE0454166. 